\title{
Novel potential perils of volunteering for clinical trials
}

\author{
Stephen B Hanauer
}

On June 11 2005, a news article in The Lancet reported on the court case of two patients with Parkinson's disease who had been enrolled in a phase II trial of glial-cell-linederived neurotrophic factor (GDNF) and were suing Amgen, the drug manufacturer. Amgen had discontinued the trial because of safety concerns identified in monkeys that had received infusions of GDNF and later developed cerebellar lesions. The patients claimed that they had reductions in tremors and no safety issues, and asked the court to prevent Amgen from withholding treatment. The report highlighted the "...lack of a suitable mechanism through which evidence from individuals can be taken into account in decision-making about experimental treatments..." and that patient choice is virtually eliminated within the realm of clinical research.

Of late, gastroenterologists and their patients have entered into similar clinical research controversies. Clinical trial reports on the benefits of natalizumab (Tysabri ${ }^{\circledR}$, Elan Corp., Ireland/Biogen Idec Inc., MA) for Crohn's disease have been published in the New England Journal of Medicine, along with reports of progressive multifocal leukoencephalopathy occurring after treatment with natalizumab. This led the manufacturers to remove the drug from the US market shortly after its approval for use in multiple sclerosis. Supplies of natalizumab for patients receiving open-label therapy for Crohn's disease, even after its apparent success in clinical trials, were also withdrawn. For those multiple sclerosis and Crohn's disease patients who were benefiting from natalizumab, most of whom had failed to respond to other agents, the option of continuing on therapy was removed.

What is even worse, is that because of their exposure to natalizumab and its potential long-latency effect on the development of progressive multifocal leukoencephalopathy, these patients are now also excluded from participating in most subsequent clinical trials. This equates to a double whammy for the
... . the

unexpected

pitfalls brought

to light by

these events

have exposed

potential

conflicts

regarding

benefits

and risks in

presenting

informed

consent...

SB Hanauer is Editorin-Chief of Nature

Clinical Practice

Gastroenterology \& Hepatology.

Competing interests

The author declared competing interests; go to

the article online for details.

www.nature.com/clinicalpractice doi:10.1038/ncpgasthep0239 patients who volunteered for these clinical trials: discontinuation of a helpful therapy and exclusion from trying other potential new therapies.

Another example of potential 'harm' from enrolling in a clinical trial pertains to patients who volunteered for early trials of infliximab (Remicade ${ }^{\circledR}$, Centocor Inc., PA/ScheringPlough Corp., NJ) for Crohn's disease. Volunteers entering the Phase I and II trials who received only a single infusion or an induction series of three infusions were not eligible for subsequent, open-label dosing. When these individuals were re-exposed to infliximab after 6-24 months, it became apparent that the initial dosing regimens were actually priming the patients for the development of immunogenicity (antibodies to infliximab), with the potential for delayed hypersensitivity reactions, acute infusion reactions, and/or eventual loss of response to the otherwise highly efficacious monoclonal antibody.

The GDNF and natalizumab examples emphasize the importance of patient choicerelated both to consent for enrolment in a trial, as well as to the need to develop mechanisms for involving patient volunteers in the decisionmaking process when the full spectrum of risks and benefits have not been fully elucidated. The natalizumab and infliximab examples have also taught us that enrolling in a trial might have unexpected negative outcomes, including exclusion from subsequent trials, or subsequent benefit once the therapy is approved by regulatory authorities. The infliximab example also reveals a potential issue regarding informed consent-enrolment in a clinical trial might preclude the volunteer from obtaining optimal benefits from the experimental agent, if and when it becomes commercially available.

I am a supporter of clinical trials but the unexpected pitfalls brought to light by these events have exposed potential conflicts regarding benefits and risks in presenting informed consent to potential volunteers. 